\title{
Importance of hyaluronan biosynthesis and degradation in cell differentiation and tumor formation
}

P. Heldin

Correspondence

P. Heldin

Ludwig Institute for Cancer Research

Biomedical Center

Box 595

S-751 24 Uppsala

Sweden

Fax: +46-18-471-4244

E-mail: paraskevi.heldin@imbim.uu.se

Presented at SIMEC 2002 (International Symposium

on Extracellular Matrix),

Angra dos Reis, RJ, Brazil,

October 7-10, 2002.

Research supported by the Swedish

Cancer Society, Konung Gustav

V:s 80-års Fond and Q-Med AB

(Uppsala).

Received December 13, 2002 Accepted February 3, 2003

\begin{abstract}
Hyaluronan is an important connective tissue glycosaminoglycan. Elevated hyaluronan biosynthesis is a common feature during tissue remodeling under both physiological and pathological conditions. Through its interactions with hyaladherins, hyaluronan affects several cellular functions such as cell migration and differentiation. The activities of hyaluronan-synthesizing and -degrading enzymes have been shown to be regulated in response to growth factors. During tumor progression hyaluronan stimulates tumor cell growth and invasiveness. Thus, elucidation of the molecular mechanisms which regulate the activities of hyaluronan-synthesizing and -degrading enzymes during tumor progression is highly desired.
\end{abstract}

Key words

- Hyaluronan

- Synthase

- Hyaluronidase

- Tumor invasion

- Extracellular matrix

- Pericellular matrix

\section{Introduction}

Hyaluronan (hyaluronic acid) is a linear glycosaminoglycan composed, on average, of 10,000 disaccharide units of glucuronic acid and $\mathrm{N}$-acetylglucosamine, with a molecular weight of $1-5 \times 10^{6}$. It belongs to the family of mucopolysaccharides that also includes heparin, chondroitin sulfate, keratan sulfate and dermatan sulfate. Hyaluronan was first described in 1934 (1), but the hyaluronan-synthesizing and -catabolizing enzymes, as well as the cell surface interacting molecules, were identified as late as in the 1990s. Hyaluronan is found in all vertebrate tissues and on the surface of certain Streptococcus and Pasteurella bacterial pathogens. Despite its simple structure, hyaluronan is involved in several biological events; it influences the hydration and physical properties of tissues and is important in the organization of pericellular and extracellular matrices through its interactions with other extracellular mac- romolecules such as aggrecan and versican. Furthermore, hyaluronan affects cell behavior, e.g., migration, proliferation and differentiation, via binding to specific cell surface receptors such as CD44. Absence of the CD44 gene virtually prevents osteosarcoma progression (2).

Alterations in the amount of hyaluronan have been documented in several diseases such as rheumatoid arthritis, inflammatory and vascular diseases, as well as cancer (3). This review will focus on the importance of hyaluronan for tumor cell proliferation and invasion.

\section{Hyaluronan-synthesizing and -degrading enzymes}

To be able to understand the roles of hyaluronan in tumor progression, increased knowledge about the mechanisms that regulate the enzymatic activities of hyaluronansynthesizing and -degrading enzymes is re- 
quired. The metabolism of hyaluronan in the mammalian body is rapid compared to other extracellular matrix components; for example, in the epidermis the half-life of hyaluronan is less than one day (4). The cells devote much energy to the synthesis of hyaluronan molecules consisting of thousands of sugars; therefore the metabolism of hyaluronan is carefully regulated in a tissuespecific manner.

The mammalian hyaluronan-synthesizing (Has) enzymes were cloned and characterized in 1996. The first Has gene cloned was from the Streptococcus pyogenes group A bacteria in 1993 using transposon mutagenesis with Tn916. Mutant cells in which the insertion of the transposon element disrupted the locus of hyaluronan synthesis were selected by visual screening of small dry colonies versus large mucoid colonies (5-7). The availability of the bacterial Has sequence led to the identification of the eukaryotic hyaluronan synthases, termed Has1, Has2, and Has3 (8-11). The mammalian hyaluronan synthase isoforms share 55-71\% amino acid sequence similarity, whereas human and mouse homologous isoforms share about $97 \%$ similarity. Based on the sequence, the Has isoforms encode plasma membrane proteins with seven membraneassociated regions and a central cytoplasmatic domain which possesses consensus sequences for phosphorylation by protein kinase C (PKC; reviewed in Ref. 12). More recently a viral Has was discovered in the genome of the chlorella virus 1 which infects chlorella-like algae in fresh water worldwide. Using the transposon mutagenesis approach, another bacterial Has has also been identified from Gram-negative Pasteurella multocida bacteria which, importantly, is about twice as large as the other Has isoforms (reviewed in Ref. 13). The evolution of the vertebrate Has family involved gene duplication and divergence. The ancestral Has in animals was duplicated to form the Has1 and Has2 lineages. A subsequent pair of duplication events resulted in the formation of Has1 and a Has-related homologue, as well as in the formation of Has2 and Has3.

Mice deficient in Has1 or Has3 activity are viable, whereas mice deficient in Has2 activity have severe developmental problems such as cardiac defects. Thus, Has2 activity is required for embryonic development (14). In all vertebrates so far studied three Has isoforms have been found and are located on different chromosomes (12). The distinct expression patterns of the Has isoforms during embryogenesis and adulthood suggest different functional roles $(15,16)$. As a tool for a more direct assessment of possible differences in the enzymatic properties of each Has isoform, our group and others have overexpressed Has genes in Chinese hamster ovary cells, COS- 1 cells or rat 3 Y1 fibroblasts $(17,18)$. These studies demonstrated that each of the three Has isoforms is capable of hyaluronan polymerization; however, they exhibit different catalytic rates for hyaluronan synthesis and produce hyaluronans of different size. In addition, the studies indicated that different cytoplasmic proteins interact specifically with each Has protein, and may have accessory or regulatory roles in hyaluronan biosynthesis (17).

The enzymes involved in hyaluronan degradation, hyaluronidases, exist in several isoforms (Hyal1, 2, 3 and PH-20) sharing about $40 \%$ similarity. They are located on human chromosomes 3p21.3 and 7q31.3. Hyal1 and Hyal 2 are found in most tissues and body fluids and are believed to act in a concerted manner to degrade hyaluronan. Very little is known about Hyal3 which is found in testis and bone marrow. $\mathrm{PH}-20$, the sperm-associated hyaluronidase, is expressed in testis and has a role in fertilization (19). All known hyaluronidases are active at acidic $\mathrm{pH}$, consistent with a lysosomal location, except PH20 which also exhibits activity at neutral $\mathrm{pH}$. For example, Hyal1, although found predominately in the circulation and urine, is active only at acidic pH. Furthermore, in 
vitro, higher Hyal activities are found in media than in cell lysates (20). It remains to be elucidated whether hyaluronidases are lysosomal and/or secreted into the extracellular matrix. In addition to their role in hyaluronan degradation, hyaluronidases may also play key roles in differentiation events; tumor cells exhibiting hyaluronidase activity induce angiogenesis of the cornea of mice (21), and more recently we have shown that $\mathrm{PH}$-20-like hyaluronidase promotes differentiation of capillary endothelial cells grown on collagen gel in a CD44-dependent manner (22). The rapid turnover of hyaluronan, which varies considerably among different tissues in the human body, raises many questions concerning the control mechanisms that modulate the expression levels and activities of hyaluronidases.

\section{Growth factor regulation of hyaluronan metabolism}

Increased levels of hyaluronan have been detected in several human cancers (for a review, see Ref. 23) and therefore we have investigated the molecular mechanisms involved in the perturbation of hyaluronan synthesis and catabolism. We have studied the regulation of Has and hyaluronidase gene expression in response to growth factors released during tumor progression. We first investigated the effects of various growth factors on the synthesis of hyaluronan in sparse and dense fibroblast cultures. Platelet-derived growth factor (PDGF-BB) had a potent stimulatory effect on hyaluronan synthesis whereas PDGF-AA was less active, suggesting that the stimulatory effect of PDGF-BB on hyaluronan synthesis is mainly mediated by its $B$-receptor. No correlation with the mitogenic effects of the growth factors was found since epidermal growth factor (EGF) and basic fibroblast growth factor (bFGF), which are as potent mitogens as PDGF-BB, had much weaker effects than PDGF-BB on hyaluronan synthesis, and since tumor growth factor beta (TGF- $\beta$ ), which inhibits cell growth, stimulated hyaluronan synthesis (24). Studies by other groups also showed a growth factor-dependent stimulation of hyaluronan synthesis in cells of mesenchymal origin $(25,26)$. The effects of the external stimuli studied on hyaluronan synthesis were mediated partly by activation of PKC and protein kinase A $(27,28)$. Further studies on the growth factor-dependent regulation of hyaluronan synthesis revealed that among the three Has isoforms, Has2 is most markedly up-regulated or suppressed in response to external stimuli in cultures of normal human mesothelial cells (16).

Notably, the growth factor regulation of hyaluronan synthesis also affected the microenvironment between the cells and the extracellular matrix. PDGF-BB and EGF, but not TGF- $\beta$, stimulated the formation of a pericellular matrix around mesothelial cells (29). However, hyaluronan synthesis and pericellular matrix formation in chick embryo limb mesodermal cells were induced by bFGF and TGF- $\beta$, but not by PDGF or EGF (30). Thus, growth factors appear to have different regulatory roles in hyaluronan synthesis and assembly in various cell types.

Very little is known about the mechanisms that regulate the activities of hyaluronidases in diseases. Studies from our laboratory using an experimental rat model of irradiation-induced lung fibrosis revealed that hyaluronidases initially induce Has activation which results in the formation of hyaluronan oligosaccharides, most likely capable of promoting the fibroproliferative response. Furthermore, the data revealed that although TGF- $\beta$, PDGF-BB and the PKCactivating tumor promoter PMA are powerful stimulators of Has activities, they exhibited differential effects on hyaluronidase activity; TGF- $\beta$ strongly inhibited hyaluronidase activities present in cell culture media, whereas PDGF-BB and PMA had no effect on hyaluronidase activities present both in media and cell fractions, as compared to 
nonstimulated cells (20). Members of the inter- $\alpha$-inhibitor family have also been shown to inhibit serum hyaluronidase activity (31).

\section{The importance of hyaluronan in tumor invasion}

Several tumor types, both epithelial and connective tissue tumors, are often enriched in hyaluronan. Human mesotheliomas, nephroblastomas and, to a somewhat lesser extent, breast carcinomas, are considered to have the highest enrichment in hyaluronan. The mean level of hyaluronan in the serum of a middle-aged healthy person is about 40 $\mu \mathrm{g} / \mathrm{ml}$. Hyaluronan levels in serum increase in various diseases, and levels above $250 \mu \mathrm{g} /$ $\mathrm{ml}$ indicate progressive disease and can be of diagnostic value. In most tumors an increase in the proportion of hyaluronan over other glycosaminoglycans is commonly seen. Most of the hyaluronan is found within the tumorassociated stromal tissue at concentrations up to $0.1 \%$ of the wet weight (32). It should also be considered that elevation of the amounts of hyaluronan in tumors in vivo may not result exclusively from increased hyaluronan synthesis. Rather, increased cellularity or decreased hyaluronidase activity may give rise to higher hyaluronan levels. Alternatively, local clearance of hyaluronan or clearance via the lymphatic tissue may be impaired in advanced tumors. However, increased hyaluronan deposition is also found during embryonic development, wound healing and regeneration. This suggests that the accumulation of hyaluronan is a common feature during tissue reconstruction. It is possible that hyaluronan, as a consequence of its physicochemical properties, creates permeable matrices within which cells can easily change shape and migrate. The function of hyaluronan accumulation in tumor tissues remains to be determined.

Immunohistochemical staining of tumor tissues for hyaluronan using a hyaluronanbinding protein isolated from bovine carti- lage proteoglycan followed by biotinylation (33) has indicated that most human solid tumors have increased amounts of hyaluronan in the stromal tissue. However, in vitro studies of tumor cell lines have revealed that not all tumor cells synthesize hyaluronan by themselves. Whereas some tumor cells such as fibrosarcoma (34) and glioma (35) produce large amounts of hyaluronan, others, such as mesothelioma and lung carcinoma cell lines, do not synthesize hyaluronan; rather these tumors secrete factors that stimulate hyaluronan synthesis by normal connective tissue cells such as mesothelial cells and fibroblasts $(36,37)$. Co-culture of fibroblasts and tumor cells also leads to stimulation of hyaluronan synthesis. Thus, most likely the host connective tissue responds to the presence of actively proliferating and migrating tumor cells.

To further explore how hyaluronan synthesized by the host connective tissue cells affects tumor invasion, we attempted to characterize its relationship to the aggressiveness of mesothelioma. We investigated the functional significance of the immediate microenvironment around mesothelial cells which is composed of hyaluronan-containing pericellular matrices that are formed in vitro in at least two different ways. One type of coat is anchored to the cell surface via hyaluronan receptors; such coats are seen around chondrocytes and their structure can be disassembled by hexasaccharides (38). The other type of coat consists of newly synthesized hyaluronan, still attached to the membrane-associated hyaluronan synthase, which is extruded into the extracellular space (29). The assembly of the latter type of coats requires serum proteins and growth factors such as PDGF-BB and EGF, and its structure differs from that found around chondrocytes in cartilage $(29,39)$. Conditioned media of mesothelioma cells possessing PDGF- and EGF-like activities induced the synthesis of hyaluronan by mesothelial cells (36) and promoted the formation of hyaluronan-con- 
taining pericellular matrices around mesothelial cells (40). It is also possible that during tumor invasion the host immune system may also play a role in the assembly of pericellular matrices through induction of hyaluronan synthesis by tumor-adjacent fibroblasts by secretion of inflammatory cytokines (41). Thus, these types of pericellular matrices may assemble and function as a protective shield around connective cells in the stromal tissue, in vivo, as a response to the release of both matrix components and growth factors by tumor cells (Figure 1A).

Several recent studies have shown that hyaluronan overexpression by various tumor cells after transfection of tumor cells with different Has isoforms enhances their malignant phenotype (42-45). In one of our experimental models we have investigated the effect of hyaluronan production on the malignant properties of mesothelioma cells.

About $70 \%$ of malignant pleura mesothelioma cases are associated with elevated amounts of hyaluronan which have been directly correlated with tumor burden. However, our in vitro studies revealed that not all mesothelioma cells obtained from various patients produce hyaluronan (36). In an effort to explore the relation between hyaluronan-producing mesotheliomas and their aggressiveness, we have compared the biological properties of the non-hyaluronan-producing mesothelioma cell line Mero-25 with those of the same cells made to produce hyaluronan by transfection with Has 2 cDNA. Our data revealed that hyaluronan-producing mesotheliomas are more aggressive and invasive than the non-hyaluronan-producing mesotheliomas (44). A possible explanation could be that hyaluronan-synthesizing tumor cells are surrounded by protective pericellular matrices which may help tumor cells evade cellular immune attack, as has been shown for glioma cells (46) (Figure $1 \mathrm{~B})$. Another possibility is that the hyaluronan synthesized by tumor cells contributes to the expansion of extracellular matrix and thereby facilitates tumor cell migration.

Recently, several contradictory findings on the functional significance of hyaluronidases in terms of tumor development have been published. In some of the studies hyaluronidase expression and activity was detected in metastatic human melanoma, glioblastoma and colon carcinoma, whereas in other studies hyaluronidase activity suppressed tumor development $(47,48)$. Based on the knowledge that Has overexpression increases tumorigenicity in the systems studied whereas Hyal overexpression increases tumor development in some experimental models and suppresses it in others, we decided to investigate the impact of Hyall and Has2 expression in a colon carcinoma model both in vitro and in vivo. These studies revealed that Has2 overexpression promotes tumorigenicity, whereas Hyal1 overexpression delays tumor development in a colon carcinoma model (49). It would therefore be interesting to study the mechanisms that regulate the activities of hyaluronan-synthesizing and -degrading enzymes during tumor progression in different tissues and organs.

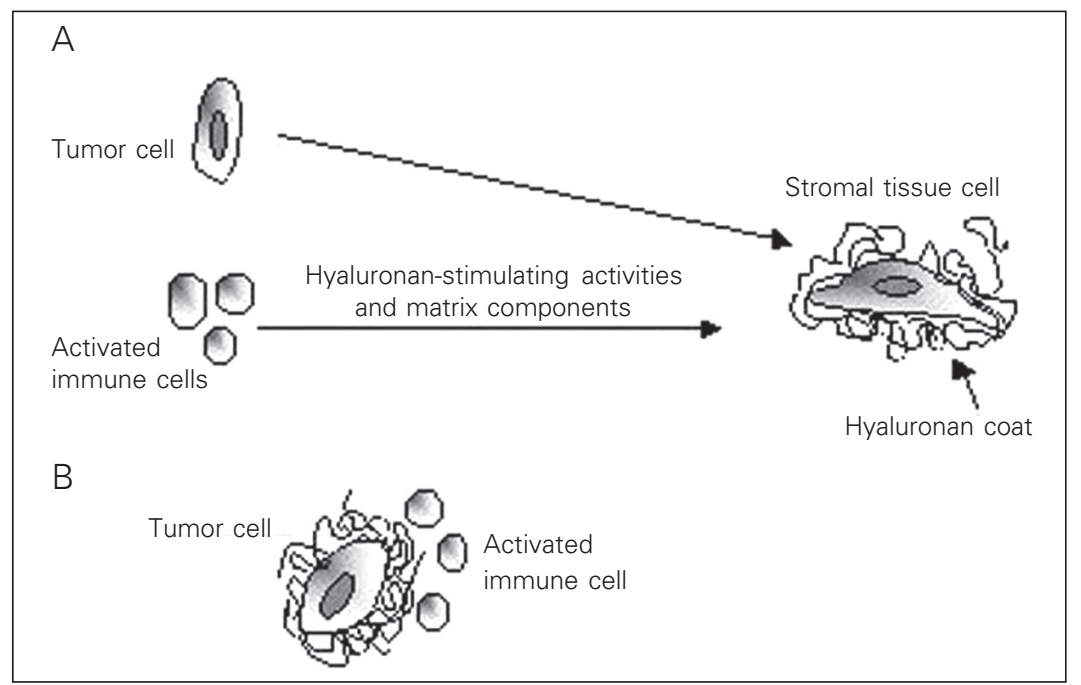

Figure 1. Hyaluronan synthesis during tumor progression. A host tumor stromal cell is surrounded by a hyaluronan-containing coat in response to hyaluronan-stimulating activities and matrix components secreted by a tumor cell and/or activated immune cells (A). A tumor cell surrounded by a hyaluronan-containing coat excludes host immune cells (B). 


\section{Future perspectives}

The cloning and characterization of eukaryotic hyaluronan-synthesizing and -degrading enzymes, as well as hyaluronan receptors, were major breakthroughs in hyaluronan research. These reagents will make it possible to raise monoclonal antibodies and construct probes suitable for immunohistochemistry and in situ hybridization, re- spectively, which can be used to increase our understanding of their roles in normal and pathological situations. For example, it will be interesting to further elucidate the roles of hyaluronan in pericellular coats and in matrices and its interactions with other molecules. Such studies should shed light on the hyaluronan-induced enhancement of tumor invasion.

\section{References}

1. Meyer K \& Palmer JW (1934). The polysaccharide of the vitreous humor. Journal of Biological Chemistry, 107: 629-634.

2. Weber GF, Bronson RT, Ilagan J, Cantor H, Schmits R \& Mak TW (2002). Absence of the CD44 gene prevents sarcoma metastasis. Cancer Research, 62: 2281-2286.

3. Laurent TC \& Fraser JRE (1992). Hyaluronan. FASEB Journal, 6: 2397-2404.

4. Tammi MI, Day AJ \& Turley EA (2002). Hyaluronan and homeostasis: a balancing act. Journal of Biological Chemistry, 277: 4581-4584.

5. DeAngelis PL, Papaconstantinou J \& Weigel PH (1993). Molecular cloning, identification, and sequence of the hyaluronan synthase gene from group A Streptococcus pyogenes. Journal of Biological Chemistry, 268: 19181-19184.

6. DeAngelis PL, Papaconstantinou J \& Weigel PH (1993). Isolation of a Streptococcus pyogenes gene locus that directs hyaluronan biosynthesis in acapsular mutants and in heterologous bacteria. Journal of Biological Chemistry, 268: 14568-14571.

7. Dougherty BA \& van de Rijn I (1994). Molecular characterization of hasA from an operon required for hyaluronic acid synthesis in group A Streptococci. Journal of Biological Chemistry, 269: 169-175.

8. Itano N \& Kimata K (1996). Expression cloning and molecular characterization of HAS protein, a eukaryotic hyaluronan synthase. Journal of Biological Chemistry, 271: 9875-9878.

9. Shyjan AM, Heldin P, Butcher EC, Yoshino T \& Briskin MJ (1996). Functional cloning of the cDNA for a human hyaluronan synthase. Journal of Biological Chemistry, 271: 23395-23399.

10. Spicer AP, Augustine ML \& McDonald JA (1996). Molecular cloning and characterization of a putative mouse hyaluronan synthase. Journal of Biological Chemistry, 271: 23400-23406.

11. Spicer AP, Olson JS \& McDonald JA (1997). Molecular cloning and characterization of a cDNA encoding the third putative mammalian hyaluronan synthase. Journal of Biological Chemistry, 272: 89578961.

12. Weigel PH, Hascall VC \& Tammi M (1997). Hyaluronan synthases. Journal of Biological Chemistry, 272: 13997-14000.

13. DeAngelis PL (1999). Hyaluronan synthases: fascinating glycosyltransferases from vertebrates, bacterial pathogens, and algar viruses. Cellular and Molecular Life Sciences, 56: 670-682.

14. Camenisch TD, Spicer AP, Brehm-Gibson T, Biesterfeldt J, Augustine ML, Calabro A, Kubalak S, Klewer SE \& McDonald JA (2000). Distruption of hyaluronan synthase-2 abrogates normal cardiac morphogenesis and hyaluronan-mediated transformation of epithelium to mesenchyme. Journal of Clinical Investigation, 106: 349-360.
15. Spicer AP \& McDonald JA (1998). Characterization and molecular evolution of a vertebrate hyaluronan synthase gene family. Journal of Biological Chemistry, 273: 1923-1932.

16. Jacobson A, Brinck J, Briskin MJ, Spicer AP \& Heldin P (2000). Expression of human hyaluronan synthases in response to external stimuli. Biochemical Journal, 348: 29-35.

17. Brinck J \& Heldin P (1999). Expression of recombinant hyaluronan synthase (HAS) isoforms in $\mathrm{CHO}$ cells reduces cell migration and cell surface CD44. Experimental Cell Research, 252: 342-351.

18. Itano N, Sawai T, Yoshida M, Lenas P, Yamada Y, Imagawa M, Shinomura T, Hamaguchi M \& Yoshida $Y$ (1999). Three isoforms of mammalian hyaluronan synthases have distinct enzymatic properties. Journal of Biological Chemistry, 274: 25085-25092.

19. Stern R \& Csoka AB (2000). Mammalian hyaluronidases. www.glycoforum.gr.jp/science/hyaluronan/HA15/HA15E.html.

20. Li Y, Rahmanian M, Widström C, Lepperdinger G, Frost GI \& Heldin $P(2000)$. Irradiation-induced expression of hyaluronan (HA) synthase 2 and hyaluronidase 2 genes in rat lung tissue accompanies active turnover of $\mathrm{HA}$ and induction of types I and III collagen gene expression. American Journal of Respiratory Cell and Molecular Biology, 23: 411-418.

21. Liu D, Pearlman E, Diaconu E, Guo K, Mori H, Haqqi T, Markowitz S, Willson J \& Man-Sun S (1996). Expression of hyaluronidase by tumor cells induces angiogenesis in vivo. Proceedings of the $\mathrm{Na}$ tional Academy of Sciences, USA, 93: 7832-7837.

22. Rahmanian $M$ \& Heldin $P$ (2002). Testicular hyaluronidase induces tubular structures of endothelial cells grown in three-dimensional collagen gel through a CD44-mediated mechanism. International Journal of Cancer, 97: 601-607.

23. Toole BP, Wight TN \& Tammi MI (2002). Hyaluronan-cell interactions in cancer and vascular disease. Journal of Biological Chemistry, 277: 4593-4596.

24. Heldin P, Laurent TC \& Heldin C-H (1989). Effect of growth factors on hyaluronan synthesis in cultured human fibroblasts. Biochemical Journal, 258: 919-922.

25. Tirone ED, Alessandris C, Hascall VC, Siracusa G \& Salustri A (1997). Hyaluronan synthesis by mouse cumulus cells is regulated by interactions between follicle-stimulating hormone (or epidermal growth factor) and a soluble oocyte factor (or transforming growth factor ß1). Journal of Biological Chemistry, 272: 4787-4794.

26. Honda A, Noguchi N, Takehara H, Ohashi Y, Asuwa N \& Mori $Y$ (1991). Cooperative enhancement of hyaluronic acid synthesis by combined use of IGF-I and EGF, and inhibition by tyrosine kinase 
inhibitor genistein, in cultured mesothelial cells from rabbit pericardial cavity. Journal of Cell Science, 98: 91-98.

27. Klewes L \& Prehm P (1994). Intracellular signal transduction for serum activation of the hyaluronan synthase in eukaryotic cell lines. Journal of Cellular Physiology, 160: 539-544.

28. Suzuki M, Asplund T, Yamashita H, Heldin C-H \& Heldin P (1995). Stimulation of hyaluronan biosynthesis by platelet-derived growth factor-BB and transforming growth factor-beta1 involves activation of protein kinase C. Biochemical Journal, 307: 817-821.

29. Heldin P \& Pertoft $H$ (1993). Synthesis and assembly of the hyaluronan-containing coats around normal human mesothelial cells. Experimental Cell Research, 208: 422-429.

30. Munaim SI, Klagsbrun M \& Toole BP (1991). Hyaluronan-dependent pericellular coats of chick embryo limb mesodermal cells: Induction by basic fibroblast growth factor. Developmental Biology, 143: $297-$ 302.

31. Mio K, Carrette O, Maibach HI \& Stern R (2000). Evidence that the serum inhibitor of hyaluronidase may be a member of the Inter- $\alpha$ inhibitor family. Journal of Biological Chemistry, 275: 32413-32421.

32. Laurent TC, Laurent UB \& Fraser JR (1996). Serum hyaluronan as a disease marker. Annual Medicine, 28: 241-253.

33. Hellström S, Tengblad A, Johansson C, Hedlund U \& Axelsson E (1990). An improved technique for hyaluronan histochemistry using microwave irradiation. Histochemical Journal, 22: 677-682.

34. Hopwood J, Fitch FW \& Dorfman A (1974). Hyaluronic acid synthesis in a cell-free system from rat fibrosarcoma. Biochemical and Biophysical Research Communications, 61: 583-590.

35. Philipson LH \& Schwartz NB (1984). Subcellular localization of hyaluronate synthetase in oligodendroglioma cells. Journal of Biological Chemistry, 259: 5017-5023.

36. Asplund T, Versnel MA, Laurent TC \& Heldin P (1993). Human mesothelioma cells produce factors that stimulate the production of hyaluronan by mesothelial cells and fibroblasts. Cancer Research, 53: 388-392.

37. Teder P, Bergh J \& Heldin P (1995). Functional hyaluronan receptors are expressed on squamous cell lung carcinoma cell line but not on other lung carcinoma cell lines. Cancer Research, 55: 3908-3914.

38. Knudson W \& Knudson CB (1991). Assembly of a chondrocyte-like pericellular matrix on non-chondrogenic cells. Role of the cell surface hyaluronan receptors in the assembly of a pericellular matrix.
Journal of Cell Science, 99: 227-235.

39. Lee GM, Johnstone B, Jacobson K \& Caterson B (1993). The dynamic structure of the pericellular matrix of living cells. Journal of Cell Biology, 123: 1899-1907.

40. Heldin P, Suzuki M, Teder P \& Pertoft H (1995). Chondroitin sulfate proteoglycan modulates the permeability of hyaluronan-containing coats around normal human mesothelial cells. Journal of Cellular Physiology, 165: 54-61.

41. Pure E \& Cuff CA (2001). A crucial role for CD44 in inflammation. Trends in Molecular Medicine, 7: 213-221.

42. Itano N, Sawai T, Miyaishi O \& Kimata K (1999). Relationship between hyaluronan production and metastatic potential of mouse mammary carcinoma cells. Cancer Research, 59: 2499-2504.

43. Kosaki R, Watanabe $K$ \& Yamaguchi $Y$ (1999). Overproduction of hyaluronan by expression of the hyaluronan synthase Has2 enhances anchorage-independent growth and tumorigenicity. Cancer Research, 59: 1141-1145.

44. Li Y \& Heldin P (2001). Hyaluronan production increases the malignant properties of mesothelioma cells. British Journal of Cancer, 85: 600-607.

45. Liu N, Gao F, Han Z, Xu X, Underhill CB \& Zhang L (2001). Hyaluronan synthase 3 overexpression promotes the growth of TSU prostate cancer cells. Cancer Research, 61: 5207-5214.

46. Gately CL, Muul LM, Greenwood MA, Papazoglou S, Dick SJ, Kornblith PL, Smith BH \& Gately MK (1984). In vitro studies on the cell-mediated immune response to human brain tumors. II. Leukocyte-induced coats of glycosaminoglycan increase the resistance of glioma cells to cellular immune attack. Journal of Immunology, 133: 3387-3395.

47. Jackson DG, Schenker T, Waibel R, Bell JI \& Stahel RA (1994). Expression of alternatively spliced forms of the CD44 extracellularmatrix receptor on human lung carcinomas. International Journal of Cancer, 8 (Suppl): 110-115.

48. Lokeshwar VB, Young MJ, Goudarzi G, lida N, Yudin Al, Cherr GN \& Selzer MG (1999). Identification of bladder tumor-derived hyaluronidase: its similarity to HYAL1. Cancer Research, 59: 4464-4470.

49. Jacobson A, Rahmanian M, Rubin K \& Heldin P (2002). Expression of hyaluronan synthase 2 or hyaluronidase 1 differentially affects the growth rate of transplantable colon carcinoma cell tumors. International Journal of Cancer, 102: 212-219. 